\title{
CMOS Active Inductor Based Voltage Controlled Oscillator
}

\author{
Dhara P Patel ${ }^{1}$, Shruti Oza ${ }^{2}$, Rajesh A Thakker ${ }^{3}$ \\ ${ }^{1}$ Electronics and Communication Engineering Department, Charotar University of Science and Technology, India \\ ${ }^{3}$ Department of Electronics and Telecommunication, Bharti Vidyapeeth Deemed University College of \\ Engineering, India \\ ${ }^{3}$ Electronics and Communication Department, Vishwakarma Government Engineering College, India
}

\begin{tabular}{l}
\hline \hline Article Info \\
\hline Article history: \\
Received Apr 1, 2017 \\
Revised Apr 20, 2017 \\
Accepted Apr 30, 2017 \\
\hline Keywords: \\
Tunable Active Inductor (TAI) \\
Voltage Controlled Oscillator \\
(VCO) \\
RF output power \\
DC to RF power efficiency \\
Phase Noise
\end{tabular}

\begin{abstract}
A Tunable Active Inductor (TAI) based Voltage Controlled Oscillator (VCO) for Radio Frequency (RF) applications ranging from $670 \mathrm{MHz}-1.53 \mathrm{GHz}$ is presented. A design of low phase noise and compact VCO is proposed. In order to lower the phase noise of VCO, its RF output power has been improved. The use of low voltage active in-ductor circuit reduces the power dissipation of VCO. The single ended CMOS active inductors with minimum number of transistors are used to consume less die area of VCO circuit. The low power dissipation of the circuit have high efficiency to generate output RF power. A supply independent variable current source tunes the VCO. The post layout design is simulated in Cadence spectreRF using TSMC $180 \mathrm{~nm}$ process libraries. The VCO circuit shows the phase noise variation from -124 to $-126 \mathrm{dBc} / \mathrm{Hz}$ and an active area of $0.0049 \mathrm{~mm}^{2}$. The VCO core circuit, excluding output buffers, consumes $10 \mathrm{~mW}$ at $1.8 \mathrm{~V}$ supply voltage.
\end{abstract}

Copyright $\odot 2017$ Institute of Advanced Engineering and Science. All rights reserved.

\section{Corresponding Author:}

Dhara P Patel,

Electronics and Communication Engineering Department,

Charotar University of Science and Technology,

CHARUSAT Campus, Off Nadiad- Petlad Highway 139, Changa, Gujarat 388421.

Email: dharapatel.ec@charusat.ac.in

\section{INTRODUCTION}

Today the emerging field of wireless communication has resulted into number of services from several megahertz to multi-gigahertz frequency bands. The performance of RF front-end mainly relies on the performance of the individual RF blocks. The frequency synthesizer is used to generate the multiple numbers of frequencies from a reference frequency. The voltage controlled oscillator is most pertinent block of frequency synthesizer. Low phase noise of VCO shows better spectral purity of frequency synthesizer. LC resonator based VCOs are widely used for RF applications instead of ring oscillators due to better phase noise performance [1]. To achieve low phase noise VCO, various techniques have been used such as switched capacitors [2] switched inductors [3], [4], and high $\mathrm{K}$ transformers [5]. But, due to the passive component like inductor, LC resonator based VCO consumes more die area. Therefore, to make the VCO compact, gyrator based active inductor technique is preferable [1]. The presented VCO shows high RF output power. The high output power VCO also reduces the driver stages of the power amplifier block of the transmitter. Subsequently, it reduces the size and power dissipation of the whole transmitter.

To tune the active inductor based VCO, various methods have been proposed as discussed in literature. In [1], voltage controlled passive resistor was used in conventional single ended cascode active inductor topology. It showed the high quality factor but the price paid was low phase noise. The tunable differential active inductor circuit was implemented in [6] and [7] to enhance the phase noise performance. But the differential configuration consumes more die area. The results of active and passive inductor based $\mathrm{VCO}(\mathrm{s})$ are also compared in literature. Proposed circuit exhibits low phase noise with consumption of less die area. 
The organization of paper is as follows. The conventional VCO topology is described in section 2. The active inductor is described in section 3 and the tuning mechanism is discussed in section 5 . The proposed VCO circuit is presented in section 4. The design and simulation results of VCO are presented in section 5. Finally, conclusions are summarized in section 6.

\section{ACTIVE INDUCTOR}

The active inductor presented in Figure 1 was used for reducing the supply noise in PC boards [8]. The single-transistor current sources in the circuit can be used for low voltage designs. The negative transconductance is realized by using M2 which is NMOS based common source configuration. Transistors M1, M3 and M4 form the positive transconductance where the current mirror contained of M3-M4 is used to invert the negative transconductance of M1, also configured in common source connection. M2 converts the voltage across $\mathrm{C} 1$ to a current and makes it to flow at the input port.

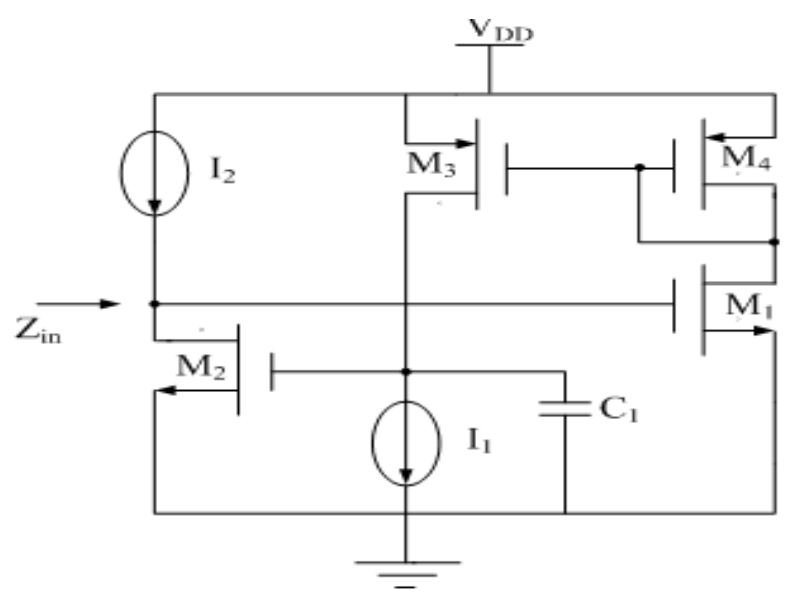

Figure 1. Low voltage active inductor [8]

The current reuse method has been used in modified low voltage active inductor as shown in Figure 2. The negative transconductance is realized by using $\mathrm{M}_{2}$ which is PMOS based common source configuration. The gate terminals of transistors $\mathrm{M}_{5}$ and $\mathrm{M}_{6}$ are tied together at node $\mathrm{V}_{\mathrm{o}}$.

\subsection{Small signal analysis}

The inductive behaviour of the modified active inductor circuit has been verified through the small signal analysis. Figure 2 shows the small signal model of proposed circuit shown in Figure 1.

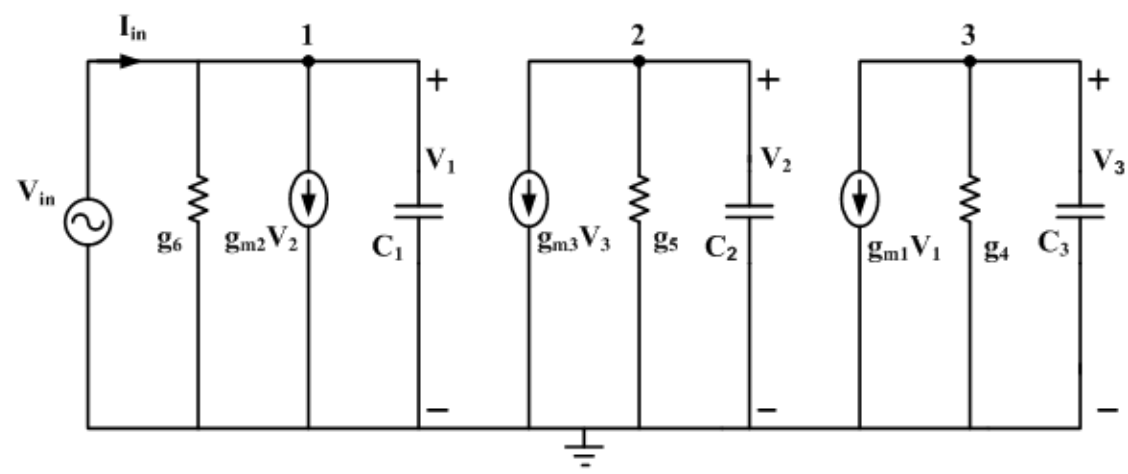

Figure 2. Small signal equivalent circuit of Figure 2

The input admittance at the input port can be expressed as, 
$Y_{\text {in }}=\frac{\left[s\left(C_{2} g_{4}+C_{3} g_{5}\right) g_{6}+C_{1} g_{4} g_{5}\right]+g_{m 1} g_{m 2} g_{m 3}}{s\left(C_{2} g_{4}+C_{3} g_{5}\right)+g_{4} g_{5}}$

From the rational function as shown in equation 1, the equivalent components as inductance (L), series resistance $\left(R_{s}\right)$, capacitance $\left(C_{p}\right)$, and parallel resistance $\left(R_{p}\right)$ can be expressed as,

$$
\begin{aligned}
& L=\frac{C_{2} g_{4}+C_{3} g_{5}}{g_{m 1} g_{m 2} g_{m 3}} \\
& R_{s}=\frac{g_{4} g_{5}}{g_{m 1} g_{m 2} g_{m 3}} \\
& C_{p}=C_{1} \\
& R_{p}=\frac{1}{g_{6}}
\end{aligned}
$$

The bode plot of the modified active inductor exhibits an inductive characteristics in the frequency range $\omega_{\mathrm{z}}<\omega<\omega_{\mathrm{p}}$.

$$
\begin{aligned}
& \omega_{z}=\frac{g_{m 1} g_{m 2} g_{m 3}}{\left(C_{2} g_{4}+C_{3} g_{5}\right) g_{6}+C_{1} g_{4} g_{5}} \\
& \omega_{p}=\frac{g_{4} g_{5}}{C_{2} g_{4}+C_{3} g_{5}}
\end{aligned}
$$

where $C_{j}=C_{g s i \mathrm{i}}(\mathrm{i}=1,2,3)$ and $g_{i}=g_{d s i}(\mathrm{j}=4,5,6)$.

Equation 2 shows that the equivalent inductance depends on the circuit parameters including $\mathrm{C}_{2}, \mathrm{C}_{3}$, $\mathrm{g}_{4}, \mathrm{~g}_{5}, \mathrm{~g}_{\mathrm{m} 1}, \mathrm{~g}_{\mathrm{m} 2}$ and $\mathrm{g}_{\mathrm{m} 3}$. It is also observed that the equivalent inductance is strongly dependent on $\mathrm{gm}_{1}, \mathrm{gm}_{2}$, and $\mathrm{gm}_{3}$. As the voltage $\mathrm{V}_{0}$ increases transconductance of $\mathbf{M}_{1}, \mathbf{M}_{2}$ and $\mathrm{M} 3$ increases and equivalent inductance decreases. In presented circuit, single tuning is provided to tune the inductance. To tune inductance and quality factor separately, capacitor $\mathrm{C}_{1}$ at node 1 can be replaced with varactor.

\section{VARIABLE CURRENT SOURCE}

One key advantage of active inductors over their spiral counterparts is the large tuning of their inductance. The gyrator-C based active inductors can be tuned either by changing the load capacitance or by varying the transconductances of the tranconductors [10]. A novel approach to use a supply independent current source to tune the active inductor circuit is proposed here. Figure 3 presents a supply independent current generator with degeneration source resistor $\mathrm{R}_{\mathrm{d}}$. The self biased circuit is supply independent but still dependent on process and temperature variation. To minimize the channel length modulation, the channel length of all the transistors in Figure 4 has been considered higher. The degenerated resistor of variable current source can be defined as,

$$
\begin{aligned}
& R_{d}=\frac{1}{\mu_{n} C_{o x}\left(\frac{W}{L}\right)_{c}\left(V_{c t r l}-V_{t h n}\right)} \\
& I_{\text {out }}=\frac{2}{\mu_{n} C_{o x}\left(\frac{W}{L}\right)_{9}} \frac{1}{R_{d}^{2}}\left(1-\frac{1}{\sqrt{K}}\right)^{2}
\end{aligned}
$$

Where $\mathrm{K}$ shows the size ration of transistors $\mathrm{M}_{9}$ and $\mathrm{M}_{10}$. The current will flow as long as the $\mathrm{K}$ is not equal to zero. In presented work, $\mathrm{K}$ is chosen as 1.5 . Over the entire controlled voltage $\left(\mathrm{V}_{\mathrm{ctrl}}\right)$ range, $\mathrm{M}_{\mathrm{c}}$ remains in triode region and transistors of NMOS and PMOS current mirrors in saturation region. With increased value of $\mathrm{V}_{\text {ctrl }}$, voltage at $\mathrm{V}_{\mathrm{o}}$ increases and provides the variable voltage at gate terminals of $\mathrm{M}_{5}$ and $\mathrm{M}_{6}$. 


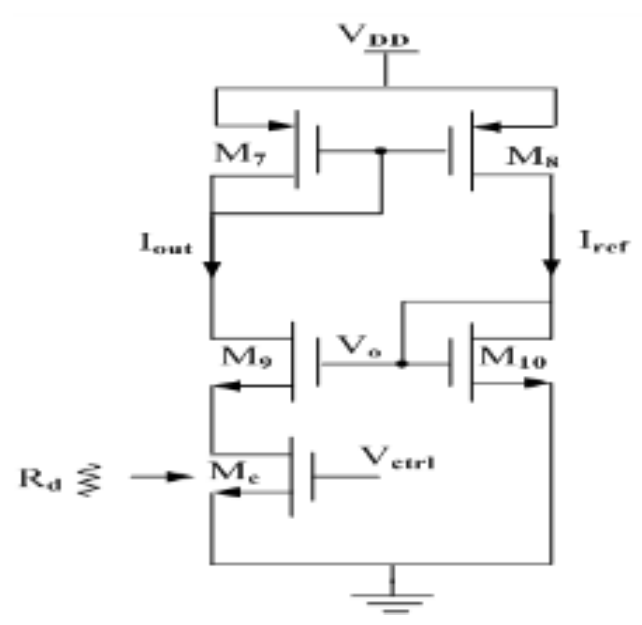

Figure 3. Variable current source [9]

\section{PROPOSED VCO CIRCUIT}

The distinct illustration of the proposed differential mode active inductor based VCO is shown in Figure 4. The modified active inductor as presented in Figure 2 has been used in VCO circuit. A variable current source controls the VCO frequency. At zero differential voltage, both switching MOSFETs of cross coupled pair remain in saturation. Furthermore, the cross coupled pair shows a small signal negative conductance that initiates the oscillations. As the control voltage (Vctrl) gradually increases, the differential voltage drives one MOSFET of cross coupled pair into triode and tuns off the other one. The FET in triode region loads the active inductor/resonator circuit.

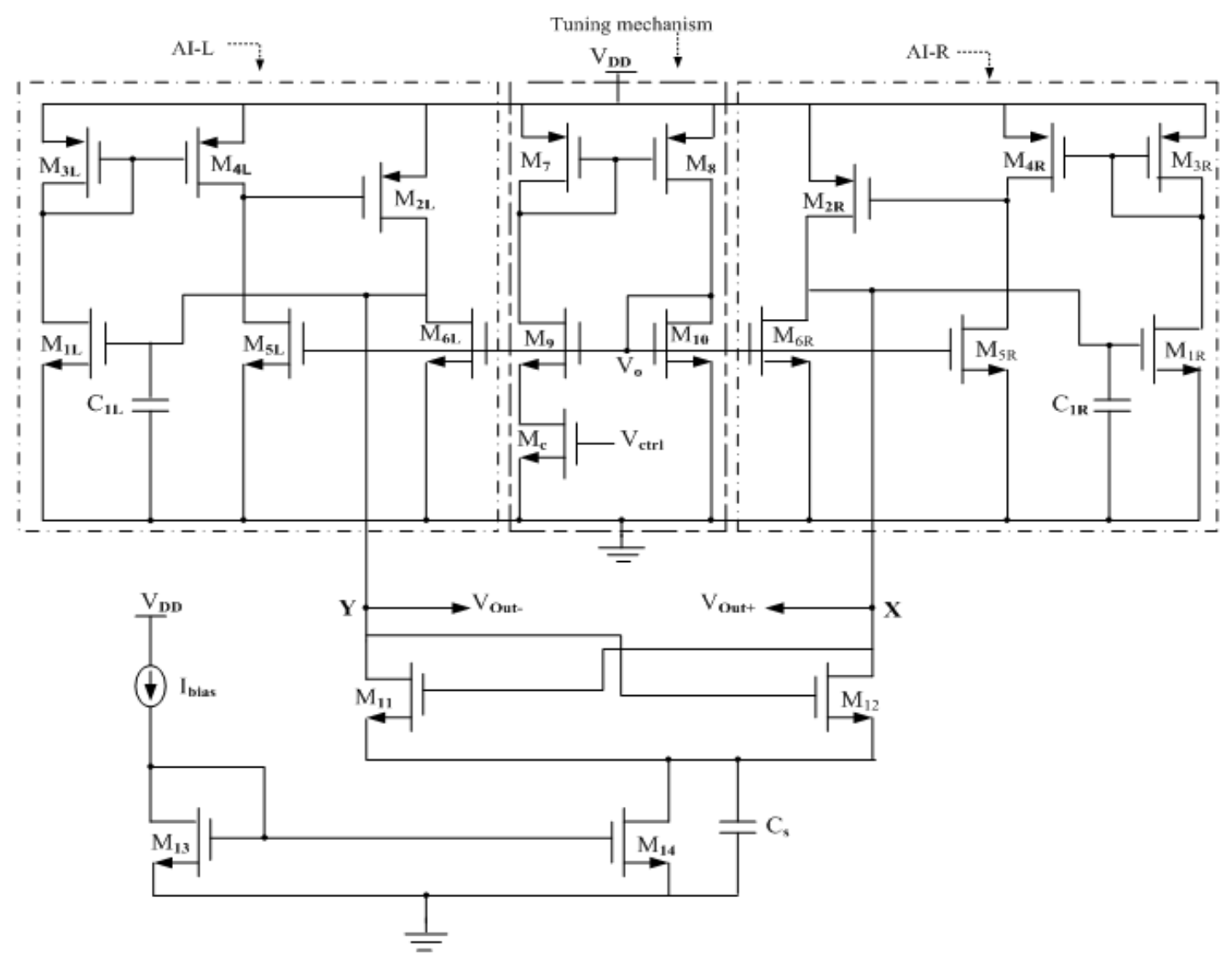

Figure 4. Proposed tunable active inductor based voltage controlled oscillator 


\subsection{VCO Start-up Conditions}

A simplified equivalent circuit of the VCO is illustrated in Figure 5. To start the oscillation, the negative conductance provided by cross coupled transistor pair $\left(\mathrm{M}_{11}\right.$ and $\left.\mathrm{M}_{12}\right)$ should be sufficiently large to compensate the loss of active inductor/-resonator circuit. To ensure about the oscillations, the negative conductance of cross coupled pair is set three times larger than the required one.

$$
\frac{1}{g_{m 11}} \approx 3 R_{p 1}
$$

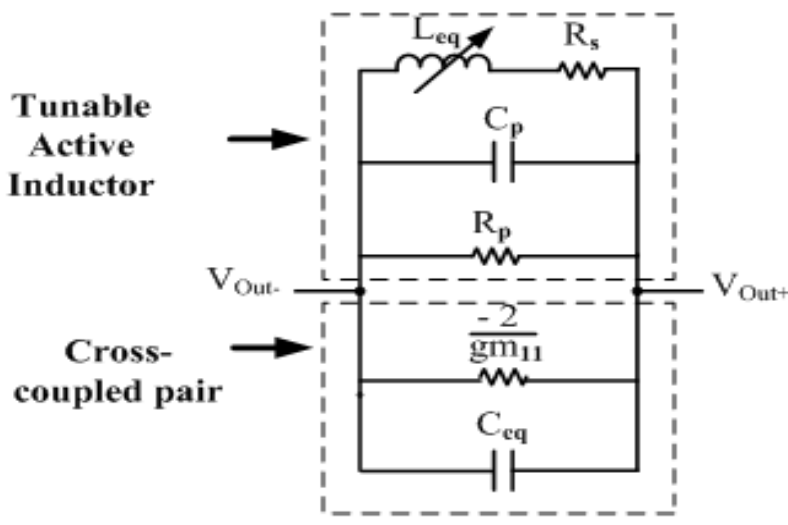

Figure 5. Simplifed VCO circuit

\subsection{VCO Frequency Tuning}

In VCO design, variable current source is used to tune the coarse frequency. As $\mathrm{V}_{\text {ctrl }}$ increases, it increases the gate voltage of $\mathrm{M}_{9}$ and $\mathrm{M}_{10}$ and hence provides the biasing to AI-L and AI-R. The resonance is measured between $\mathrm{X}$ and $\mathrm{Y}$. Variable capacitor at node $\mathrm{X}$ and $\mathrm{Y}$ can be used for fine tuning of VCO. The operating oscillation frequency can be calculated as,

$$
\omega_{o s c}=\frac{1}{\sqrt{L\left(C_{g s 12}+C_{p}\right)}}
$$

\subsection{Phase Noise}

Phase noise defines the spectral purity of the VCO output frequency. It is a important design param eter of VCO for RF applications. From Leeson's model, phase noise can be defined as,

$$
£_{(\Delta \omega)}=10 \log \left[\frac{2 k T}{P_{s i g}}\left(\frac{\omega_{0}}{2 Q \Delta \omega}\right)^{2}\right]
$$

Where $E_{(\Delta \omega)}$ the single ended spectral noise density at a frequency is offset $\Delta \omega$ away from the fundamental frequency $\omega_{0}$ and $P_{\text {sig }}$ is the power of signal [11]. Eq. (13) shows that a very efficient way to reduce the phase noise of VCO is increasing the signal power.

\subsection{DC-to-RF Power Efficiency}

The RF to DC power ratio of VCO is expressed as,

$\eta=\frac{P_{R F}}{P_{D C}}$

Low power dissipation of active inductor helps to reduce the power dissipation of VCO. Subsequently,high DC to RF power efficiency has low level of power dissipation.

\section{SIMULATION RESULTS}

The layout of proposed core VCO circuit is shown in Figure 6. VCO transistor dimensions are shown in Table 1. The post layout simulations of proposed VCO circuit are presented. Figure 7 and Figure 8 
depict the variation of VCO output frequency and phase noise variation as a function of Vctrl. VCO frequency tuning range is found as $78 \%$. Due to the higher output impedance at X and Y, VCO output power is found between $+21 \mathrm{dBm}$ to $+22 \mathrm{dBm}$. Using Equation 14, the DC-to-RF power efficiency is obtained as $15.84 \%$ which is observed highest among reported VCO circuits. Along with the higher power efficiency, the proposed circuit shows the output swing of $+/-600 \mathrm{mV}$.

Table 1. Circuit Parameters of VCO

\begin{tabular}{cc}
\hline Transistors & $\mathrm{W} / \mathrm{L}(\mu \mathrm{m} / \mu \mathrm{m})$ \\
\hline $\mathrm{M}_{1 \mathrm{~L}}, \mathrm{M}_{4 \mathrm{~L}}, \mathrm{M}_{2 \mathrm{~L}}, \mathrm{M}_{1 \mathrm{R}}, \mathrm{M}_{4 \mathrm{R}}, \mathrm{M}_{2 \mathrm{R}}, \mathrm{M}_{9}$ & $15 / 0.18$ \\
$\mathrm{M}_{3 \mathrm{~L}}, \mathrm{M}_{3 \mathrm{R}}, \mathrm{M}_{7}, \mathrm{M}_{8}, \mathrm{M}_{10}$ & $10 / 0.18$ \\
$\mathrm{M}_{5 \mathrm{~L}}, \mathrm{M}_{6 \mathrm{~L}}, \mathrm{M}_{5 \mathrm{R}}, \mathrm{M}_{6 \mathrm{R}}$ & $5 / 0.25$ \\
$\mathrm{M}_{\mathrm{c}}$ & $8 / 0.25$ \\
$\mathrm{M}_{11}, \mathrm{M}_{12}$ & $40 / 0.18$ \\
$\mathrm{M}_{13}, \mathrm{M}_{14}$ & $10 / 0.25$ \\
\hline
\end{tabular}

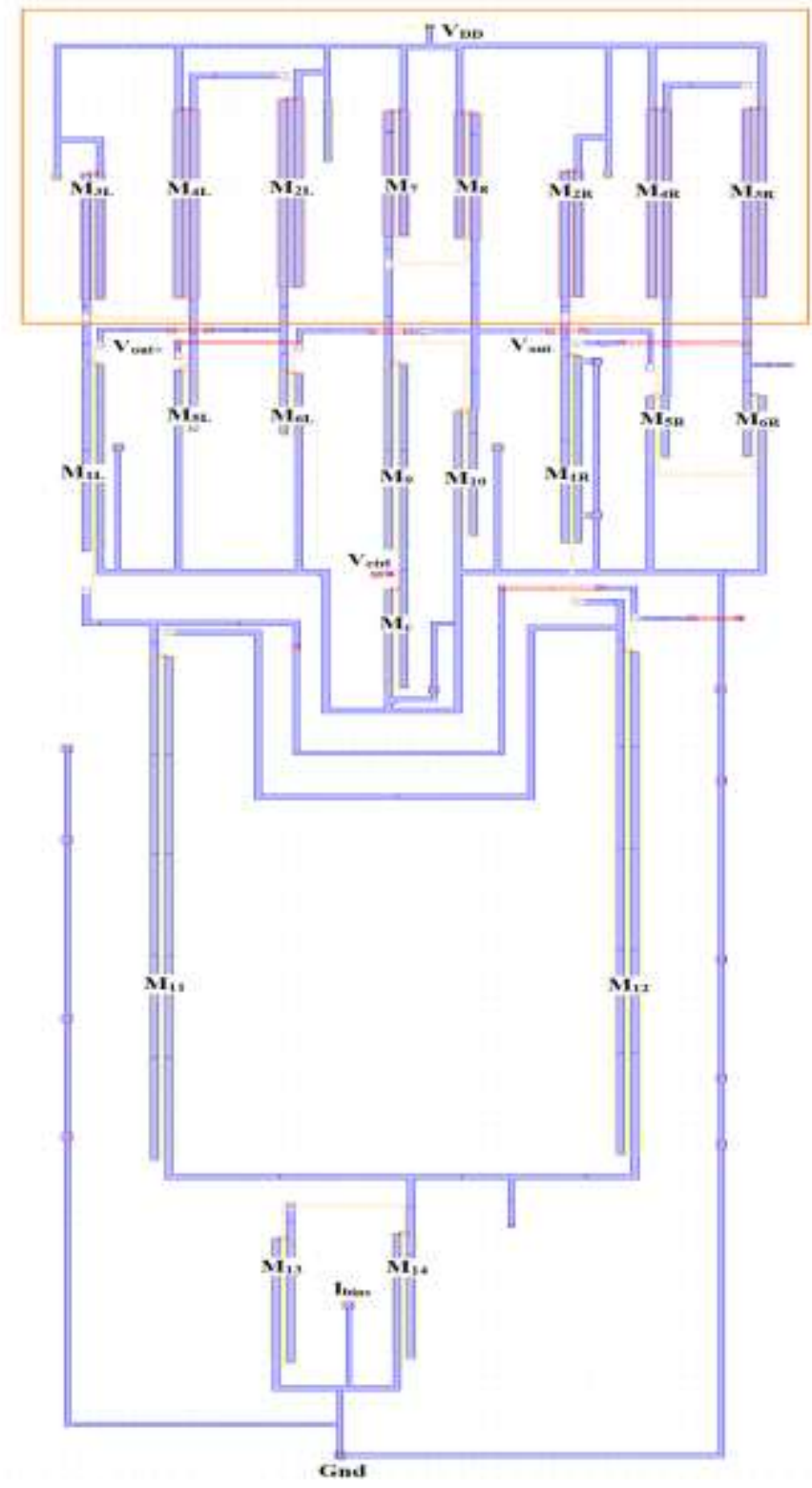

Figure 6. Proposed VCO layout 


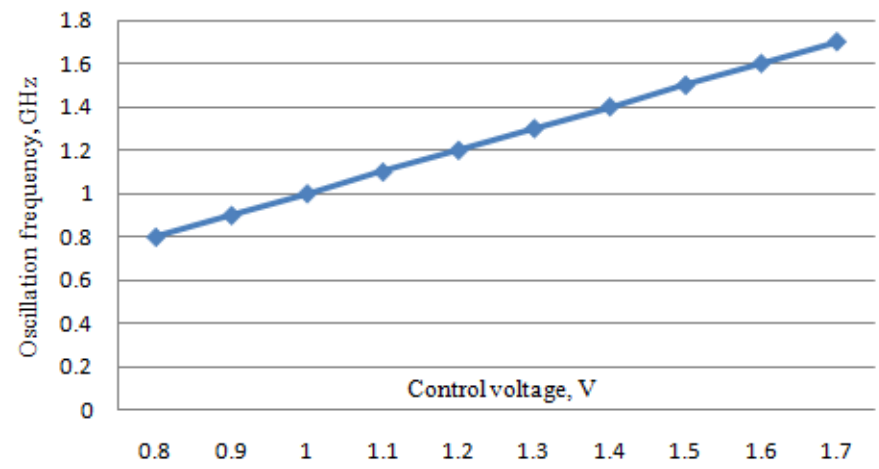

Figure 7. VCO output frequency as a function of control voltage $\mathrm{V}_{\text {ctrl }}$

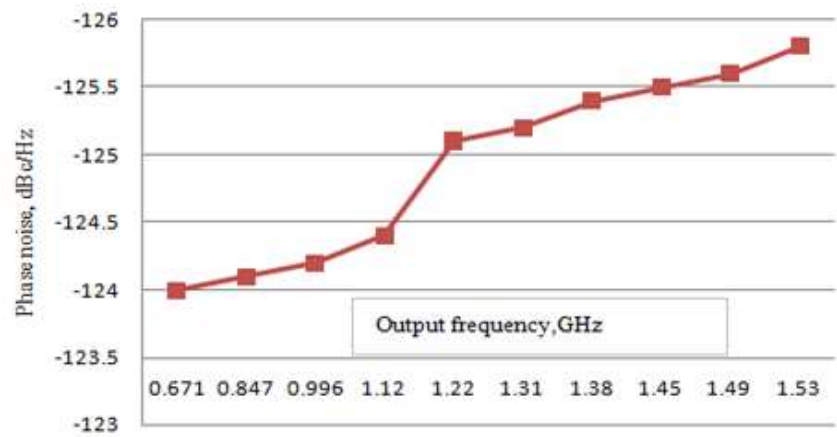

Figure 8 . VCO phase noise (at $1 \mathrm{MHz}$ offset frequency) as a function of control voltage $\mathrm{V}_{\text {ctrl }}$

Table 2 shows measured parameters of presented active inductor based VCO as well as that those of other reported work. The phase noise at $1 \mathrm{MHz}$ frequency offset is significantly lesser in the proposed VCO. It also demonstrates better DC to RF power efficiency and consumes less area.

Table 2. VCO Performance and Comparison

\begin{tabular}{|c|c|c|c|c|c|}
\hline & This work & R.Mukho 2005 [1] & L.Lu 2006 [6] & $\mathrm{Xu} 2011$ [7] & Roy 2015 [12] \\
\hline & $0.18-\mu \mathrm{m}$ & $0.18-\mu \mathrm{m}$ & $0.18-\mu \mathrm{m}$ & $0.18-\mu \mathrm{m}$ & $0.18-\mu \mathrm{m}$ \\
\hline Process & CMOS & CMOS & CMOS & CMOS & CMOS \\
\hline $\begin{array}{l}\text { Tuning range } \\
(\mathrm{GHz})\end{array}$ & $0.6-1.53$ & $0.5-2$ & $0.5-3$ & $0.83-3.72$ & $0.98-1.1$ \\
\hline $\begin{array}{l}\text { Phase noise } \\
(\mathrm{dBc} / \mathrm{Hz})\end{array}$ & $\begin{array}{c}-124 \sim-126 \\
@ 1 \mathrm{MHz} \\
\text { offset }\end{array}$ & $\begin{array}{c}-79 \sim-90 \\
@ 1 \mathrm{MHz} \\
\text { offset }\end{array}$ & $\begin{array}{c}-101 \sim-118 \\
\text { @ } 1 \mathrm{MHz} \\
\text { offset }\end{array}$ & $\begin{array}{c}-104 \sim-109 \\
\text { @ } 1 \mathrm{MHz} \\
\text { offset }\end{array}$ & $\begin{array}{c}-125 \\
\text { @ } 1 \mathrm{MHz} \\
\text { offset }\end{array}$ \\
\hline $\begin{array}{l}\text { Power dissi- } \\
\text { pation }(\mathrm{mW})\end{array}$ & 10.2 & 13.8 & $6 \sim 28$ & 13 & 48 \\
\hline $\begin{array}{c}\text { DC-RF } \\
\text { power efficiency }(\%)\end{array}$ & 15.84 & 0.09 & 0.14 & 6.25 & 5.7 \\
\hline Area $(\mathrm{mm})^{2}$ & 0.0049 & 0.09 & 0.045 & 0.108 & 0.455 \\
\hline Technique & Active Inductor & Active Inductor & Active Inductor & Active Inductor & $\begin{array}{l}\text { Passive } \\
\text { Inductor }\end{array}$ \\
\hline
\end{tabular}

\section{CONCLUSION}

A conventional low voltage active inductor circuit has been modified to use the current reuse technique. A new mechanism to tune the active inductor is used. Low voltage active inductor and less number of transistors consume less power and enhance the output RF power. Subsequently, phase noise of VCO is significantly decreased compared to passive inductor based VCO. 


\section{REFERENCES}

[1] R.Mukhopadhyay, Y. Park, P. Sen, "Reconfigurable RFICs in Si-based Technologies for a Compact Intelligent RF front-end", IEEE Transactions on Microwave Theory and Techniques, pp. 88-93, vol. 53.1, January-2005.

[2] A D. Berny, A M. Niknejad and R G. Meyer, "Wideband Low-Phase-Noise CMOS VCO ", IEEE Custom Integrated Circuits conference, pp 555-558, September 2003.

[3] F.Herzel, H.Erzgraber nad N. Ilkov, "New Approach to fully Integrated CMOS LC Oscillators with a very large tuning range ", IEEE Custom Integrated Circuits Conference, pp 573-576, May 2000.

[4] Z. Li and K.K O, "1-V low phase noise multi-band CMOS voltage controlled oscillator with switched inductors and capacitors ", IEEE Radio Frequency Integrated Circuits Symp. Dig., pp 467-470, June 2004.

[5] Safarian, Zahra and Hossein Hashemi, "Wideband Multi-Mode CMOS VCO Design Using Coupled Inductors", IEEE Transactions on circuits and systems, pp. 1830-1843, vol. 56, August-2009.

[6] L. H. Lu, H. H. Hsieh and Y. T. Liao, "Wide Tuning-Range CMOS VCO with a Differential Tunable Active Inductor", IEEE Transactions on Microwave Theory and Techniques, pp. 3462-3468, vol. 54, September-2006.

[7] Xu, Jiangtao, Carlos E. Saavedra and Guican Chen, "An Active Inductor Based VCO with wide Tuning Range and High DC-to-RF power Efficiency", IEEE Transactions on circuits and systems, pp 462-466, vol. 58, August, 2011.

[8] Taparia, Ajay, Bhaskar Banerjee, and Thayamku- langara R. Viswanathan, "Power Supply Noise Reduction Using Active Inductors in Mixed-Signal Systems", IEEE Transactions on Very Large Scale Integration (VLSI) Systems, pp. 1960- 1968, vol. 19, November-2011.

[9] Swei, Yuwen, "Supply Independent Biasing Circuit", U.S. Patent Application No. 13/237,177, September 2011.

[10] Fei Yuan, CMOS Active Inductors and Transformers Principle, Implementation and Applications,

[11] Springer, 2008, pp 21-99.

[12] A.Thanachayanont and A. Payne, "CMOS Floating Active Inductor and its Applications to Bandpass Filter and Oscillator Designs ", IEEE Proc.-Circuits Devices Syst., pp. 42-48, vol. 147, February 2000.

[13] Palash roy, Debasis dawn, "High Power and High Efficiency CMOS VCO", Microwave and optical technology letters, pp 2437-2441, vol. 57, October 2015.

[14] Kia Hojjat babaei, A'ain, Abu Khari, "Wide Tuning- Range CMOS VCO Based on a Tunable Active Inductor", International Journal of Electronics, pp 88-97, vol. 101, February 2014.

[15] B. Min and H. Jeong, "5 GHz CMOS LC VCOs with Wide Tuning Ranges ", IEEE Microwave Wireless Components Letters, pp 336-338, vol. 15, May 2005.

[16] R.S Rana, X. D Zhou and Y Lian, "An Optimized 2.4 GHz CMOS LC tank VCO with $0.55 \%$ V Frequency Pushing and 516 MHz Tuning Range", IEEE Int. Circuits Syst. Symp, pp 4811-4814, May 2005.

[17] B.De Muer, N Itoh, M. Borremans and M. Steyaert, "1.8 GHz Highly Tunable Low Phase Noise CMOS VCO ", IEEE Custom Integr. Circuits Conf., pp 585-588, May 2000.

[18] Emad Hegazi, "A Filtering Technique to Lower LC Oscillator Phase Noise", IEEE journal of solid state circuits, pp. 1921-1930, Vol. 36, December 2001.

[19] Babak soltanian, "Tail current Shaping to Improve Phase Noise in LC Voltage Controlled Oscillator", IEEE transactions on solid state circuits, pp.1792- 1802, vol.41, August 2006.

[20] Ler, Chun-Lee, Abu Khari Bin A'ain and Albert Victor Kordesch, "CMOS Active Inductor Linearity Improvement Using Feed-Forward Current Source Technique", IEEE transactions on microwave theory and techniques, pp 19151924, vol. 57, August- 2009.

[21] Roy, Palash, and Debasis Dawn. "A High Power and High Efficiency CMOS VCO." Microwave and Optical Technology Letters 57.10 (2015): 2437-2441. 